\title{
Voxel classification based airway tree segmentation
}

\author{
Pechin Lo ${ }^{a}$ and Marleen de Bruijne ${ }^{a, b}$ \\ ${ }^{a}$ Image Group, Department of Computer Science, University of Copenhagen, Denmark; \\ ${ }^{b}$ Biomedical Imaging Group, Departments of Radiology and Medical Informatics, Erasmus MC \\ - University Medical Center Rotterdam, The Netherlands
}

\begin{abstract}
This paper presents a voxel classification based method for segmenting the human airway tree in volumetric computed tomography (CT) images. In contrast to standard methods that use only voxel intensities, our method uses a more complex appearance model based on a set of local image appearance features and $\mathrm{K}^{\text {th }}$ nearest neighbor $(\mathrm{KNN})$ classification. The optimal set of features for classification is selected automatically from a large set of features describing the local image structure at several scales.

The use of multiple features enables the appearance model to differentiate between airway tree voxels and other voxels of similar intensities in the lung, thus making the segmentation robust to pathologies such as emphysema. The classifier is trained on imperfect segmentations that can easily be obtained using region growing with a manual threshold selection. Experiments show that the proposed method results in a more robust segmentation that can grow into the smaller airway branches without leaking into emphysematous areas, and is able to segment many branches that are not present in the training set.
\end{abstract}

Keywords: Segmentation, classification, airways, pulmonary CT

\section{INTRODUCTION}

Chronic obstructive pulmonary disease (COPD) is currently among the leading causes of disability in the world with a trend of going to be even more widespread in the future. ${ }^{1,2}$ It has been shown that computed tomography $(\mathrm{CT})$ has the potential to allow quantification of various COPD related diseases, for example emphysema and bronchitis. It is known that $\mathrm{CT}$ has various limitations, such as concerns of radiation exposure to the subject and which measurements should be used to assess small changes in lung structure. Nevertheless, CT is currently the only readily accessible, relatively noninvasive technique that is capable of providing quantitative structural data in vivo. ${ }^{3}$

Various studies focusing on analysis of airways in CT, mainly the measurement of airway wall thickness, have been reported. ${ }^{4-6}$ Airway tree segmentation plays a critical role in these studies, offering a starting point for conducting measurements on the airways. As the lungs are anatomically subdivided into subregions based on the structure of the airway tree, airway tree segmentation is also important for tasks such as lobe or lobule segmentation. ${ }^{7}$ Another possibility is to use characteristic points of the segmented airways, e.g. bifurcation points, as landmarks to guide registration process resulting in a better and more natural transformation for disease progression monitoring. ${ }^{8}$

A significant amount of work has been put into finding both semi-automated and automated solutions for the segmentation of airway trees in CT images. ${ }^{9-14}$ Current approaches mainly apply different variants of the region growing algorithm on the image intensity, with the assumption that the airway tree is a dark object surrounded by a bright background. The challenge of airway segmentation arises when this assumption does not hold in some region, either due to noise, reconstruction artifacts or pathologies. This results in 'explosion' or 'leaking' of the region growing process, in which surrounding lung regions are wrongly labelled as part of the airway tree. This is especially apparent for pathological cases such as emphysema, where the pathological lung tissues have exactly the same intensity as the airways. This makes it impossible to differentiate airways and lung tissues using image intensity alone. Various strategies have been applied to detect the occurance of these explosions

pechin@diku.dk 
and stopping the region growing process when explosion occurs. The strategies used generally involve heuristic rules based on geometrical properties of the region labelled. Some examples of geometrical properties used are volume of the regions segmented, ${ }^{9,10}$ radius of propagation fronts, ${ }^{13}$ and local neighborhood structures. ${ }^{14}$ There are also methods that use geometrical properties within a volume of interest that is derived using anatomical knowledge of the airway tree, such as area cross sections ${ }^{12}$ and topology of a thinned structure. ${ }^{13}$

In this paper, we propose a voxel classification based approach for segmenting the human airway tree. Region growing is performed on the posterior probabilities obtained from a classifier instead of the image intensities. Rather than making the decision whether a voxel belongs to lung or airway based on the intensity alone, a number of local image descriptors are used to make this decision with a classifier. Similar approaches that are applied on extracting general lung structures ${ }^{15,16}$ have shown promising results. By using different local image descriptors, the classifier is thus able to better differentiate between airway voxels and other voxels in the lung that may have similar intensities. Therefore it is less likely for the region growing algorithm to leak into surrounding structures when applied on the posterior probability from the classifier. Smaller branches can also be more reliably segmented, as there is no need to terminate the region growing process upon detection of an explosion.

A major drawback of classification approaches is that the labelled data set needed for the training process is not always easy to obtain. In the case of a $3 \mathrm{D}$ tree structure as complex as the airway tree, reliable manual segmentations are even harder to come by. In this work, we investigate the use of imperfect segmentations that can be easily obtained using a minimal amount of user interaction. These imperfect segmentation may only have an incomplete airway tree individually. By having a collection of imperfect segmentations, chances are that we may be able to obtain enough training samples that can describe the whole airway tree. Therefore the end result of a method trained using these segmentations may be on average much better than the individual segmentations.

We start by explaining the underlying concept of the proposed method in Sec. 2, which includes the generation of the data set for training purpose and the way the classifier is trained. Details on the parameters used for conducting the experiments along with the results are presented in Sec. 3. A discussion on the results obtained is given in Sec. 4. We conclude by giving a few remarks and a general summary of this paper in Sec. 5 .

\section{METHODOLOGY}

A detailed description of the methodology used to obtain a surrogate ground truth for the training of the proposed voxel classification method is first presented in Sec. 2.1. It is then followed by a description of the lung, trachea and main bronchus segmentation in Sec. 2.2 that are performed before the extraction of training samples in Sec. 2.3. Details on how voxels are separated into airway and non-airway class, and how training samples are extracted from both classes are given in Sec. 2.3. The type of classifier used and method for training it is given in Sec. 2.4. Finally the way the classifier is used in the overall segmentation framework is presented in Sec. 2.5.

\subsection{Surrogate Ground Truth}

One of the drawbacks with the voxel classification approach is that there is a need for labelled training data, which is something that is not easily available, especially for airway trees. Instead of using high quality manual annotations of the airway trees for training, segmentations from an interactive region growing algorithm are used instead. This segmentation will also be used as a surrogate ground truth for the validation of the proposed method. For simplicity, we will refer to the surrogate ground truth as ground truth in the following sections. An additional segmentation, which we refer to as 'exploded' segmentation, is also obtained and used in the training process to compensate for the imperfectness of this ground truth.

The interaction required from the user for the interactive region growing algorithm is limited to selecting a suitable intensity threshold. The seed point within the trachea that is required by the algorithm is obtained automatically using a trachea searching algorithm. An explosion control based on volume monitoring ${ }^{10}$ is also implemented into the interactive region growing algorithm. Upon detection of an explosion the interactive region growing algorithm warns the user of the possibility of an explosion and lets the user makes the decision whether 

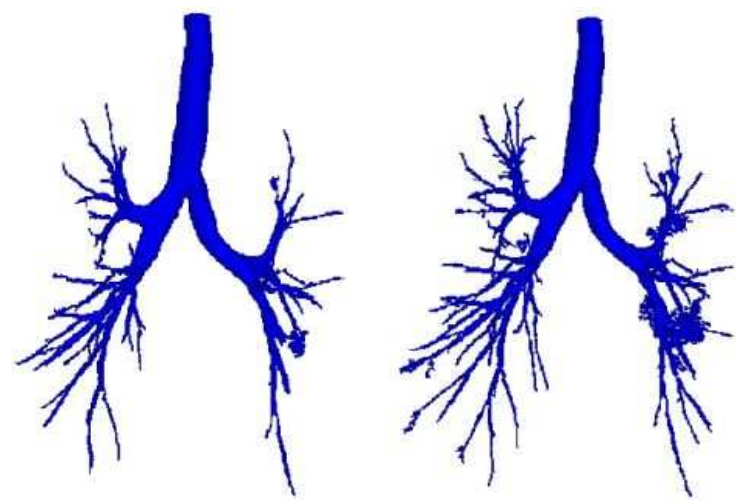

Figure 1. An example of the ground truth (left) and exploded segmentation (right).

to continue or stop the algorithm. Upon selection of continuing, the algorithm will proceed in a discrete step. This feature allows the user to fine tune the amount of additional airways segmented and the degree of explosion.

The criteria for segmenting the ground truth is to obtain as much airway as possible without leakage. In the case where leakage cannot be avoided, as little leakage as possible is preferred.

The exploded segmentation is done in such a way that most airway branches will be extracted, along with a certain degree of explosion. The reason for the inclusion of this second segmentation is to exclude potential airway points that were missed in the ground truth from the non-airway class in the training samples. Therefore, reducing the errors caused by the imperfectness of our ground truth. An example of both ground truth and exploded segmentation is shown in Fig. 1.

The segmentation procedure to obtain both the ground truth and the exploded segmentation from a CT image series takes only a couple of minutes of interaction time, wherein the user tries a number of different threshold levels for the region growing process.

\subsection{Lung, Trachea and Main Bronchus Segmentation}

A lung segmentation based on thresholding and smoothing ${ }^{17}$ is first used to segment the lung fields in the image. This lung segmentation is meant for defining the region of interest of non-airway regions in the training process.

The trachea and the main bronchus are generally situated outside the lung fields. As these regions are surrounded by tissues that have a high contrast as compared to the airways, they can be easily and reliably segmented using intensity. In order to not waste computing resources on these relatively simple structures and to make the classifier more specific to the small airways and their surroundings, trachea and main bronchus are therefore processed separately.

A fast marching algorithm that is capable of detecting bifurcations ${ }^{11}$ is used for this purpose. Two passes of the algorithm with a hole filling process in between, are used to extract the trachea and the main bronchus. The first pass is applied on the image itself using a predefined threshold of $t_{\text {airway }}$, which is chosen high enough such that noise within the airways can be included into the segmentation.

The radius of the propagating front is monitored at different time steps. Bifurcation and explosion are detected by taking the ratio between the radius at current time step and the radius of the initial front. When the ratio is larger than $\alpha_{\text {bifurcation, }}$, it is said that the front is reaching a bifurcation, and connected component analysis is then performed at every time step after this to detect the actual point where the bifurcation occurs. This detection of bifurcation also enables the algorithm to assign different labels to individual branches. The explosion is detected via a second threshold $\alpha_{\text {explode }}$, where an explosion is said to occur when the ratio exceeds this threshold.

Due to the presence of noise and reconstruction artifacts within the airways, the labels assigned by the first pass may contain errors, which is the reason for introducing a second pass. To remove noise from the first pass, a hole filling algorithm is applied to the segmented airways before proceeding to the second pass. The parameters 
for the second pass are the same as for the first pass, only this time it is applied on the region marked by the first pass and the hole filling process. This extracted trachea and main bronchus are then excluded from the ground truth airways during training in the next section.

\subsection{Training samples}

The training samples are divided into two classes, airway and non-airway class. Voxels belonging to the airway classes are voxels labelled in the ground truth, excluding the trachea and main bronchus.

We limit the non-airway class to only voxels that are within the lung fields, indicated by the regions segmented by the lung segmentation algorithm. As the classifier is to be used in a region growing framework, we are only interested in the regions around the airway tree. To limit the training samples to regions around the airway tree, dilation is first applied on the the voxels belonging to the airway class, and then non-airway voxels that are outside of this dilated region are excluded. It is known that airway voxels will never exceed a certain intensity. Therefore voxels in the non-airway class that have intensities higher than a threshold $t_{\text {air }}$ are automatically excluded from the non-airway class. These voxels are also excluded automatically by the segmentation process later on. Finally, non-airway class voxels that coincide with the 'exploded' segmentation are excluded. This is to prevent airway regions that are missed by the ground truth from being misclassified as non-airway during the training process, which would prevent such regions from being labelled as airways in the segmentation.

To prevent the classifier from training on redundant training sample, only a percentage $C$ of the voxels belonging to the airway class are extracted and used as training samples. The same amount of voxels is also extracted from the non-airway class.

As airway branches differ in size, using random sampling on airway class voxels for extracting the training samples will introduce a bias towards the larger airway branches. This bias will cause the classifier to focus more on the larger branches, rather than on the more important small branches. To overcome this problem, a distance transform operation is introduced before the sampling process. In the distance transform operation, for each airway voxel, the distance along the airway to the nearest main bronchus voxel is calculated. The airway class voxels are then separated evenly into $N$ bins based on their distance. A fixed number of samples $C V / N$, where $V$ is the total number of airway class voxels in the current airway tree, are sampled from each bin via random sampling. This sampling is started from the bin with voxels that have the largest distance. In the case where the number of samples needed is larger than the number of voxels in the current bin, the extra samples needed are extracted from the next bin. The non-airway voxels are extracted using random sampling.

\subsection{Classifier Training}

A KNN classifier is used as the classifier for the proposed method. A fast implementation based on approximate nearest neighbor searching ${ }^{18}$ is used for the KNN classifier, with the error eps set to zero to turn off the approximation part of the algorithm.

An initial set of local image descriptors is first decided and is used to compute the features for each training sample. As performance of KNN classifier depends on the quality of the features given, sequential forward floating feature selection ${ }^{19}$ is used to select an optimal subset of features from the initial set. This optimal subset of features is determined by maximizing the area under the receiver operating characteristic (ROC) curve of the KNN classifier during the feature selection process.

To perform the feature selection process, the training samples are further separated into two sets, a training set used to construct a KNN classifier for each of the feature combinations, and a validation set to evaluate the performance of the combinations. Again when separating samples belonging to the airway class, care is taken such that the separation, which is done in a random manner, is perform on each of the $N$ bins separately.

The feature selection process is stopped when a maximum of $F$ features are selected. Along with the optimal set of features, the optimal threshold $t_{\text {opt }}$ obtained from the ROC curve of the optimal feature set is also stored. This optimum threshold is later used as decision boundary for the KNN classifier.

The final KNN classifier used in the segmentation process is constructed using the optimum features from all the training samples. 


\subsection{Segmentation Framework}

The segmentation framework of the proposed method starts by segmenting the trachea and main bronchus. This segmentation is done using the method described in Sec. 2.2. With the trachea and main bronchus segmented, the voxel classification based region growing algorithm is then started, using the segmented main bronchus as initial seed points.

In the classification bases region growing algorithm, voxels with an intensity higher than $t_{\text {air }}$ are automatically rejected. Features from the optimal set of a voxel are first computed and used as input for the KNN classifier. Posterior of the voxel belonging to the airway class can then be obtained from the KNN classifier by computing $K_{a} / K$, where $K_{a}$ is the number of nearest neighbors belonging to the airway class and $K$ is the total number of nearest neighbor returned by the KNN classifier. As mentioned in the previous section, rather than using a maximum a posteriori based cut off point of 0.5 , the optimal threshold $t_{\text {opt }}$ obtained from the ROC curve analysis is used to determined whether a voxel belongs to airway or non-airway. The region growing algorithm stops if the posterior of a voxel is lower than $t_{\text {opt }}$.

\section{EXPERIMENT AND RESULTS}

Cross-validation experiments were performed on 20 low dose CT images of 20 randomly selected subjects. Results from an intensity based region growing algorithm are presented along with results from the proposed method for comparison purpose.

\subsection{Parameter settings}

In segmentation of trachea and main bronchus, the parameters $t_{\text {airway }}, \alpha_{\text {bifurcation }}$ and $\alpha_{\text {explode }}$ are set to $-900 \mathrm{HU}, 1.3$, and 3 respectively. The percentage $C$ of voxels in an airway tree where training samples are to be extracted from is set to $40 \%$. A threshold value of $-380 \mathrm{HU}$ is selected for $t_{\text {air }}$. The number of bins $N$ used to separate the airway class voxels based on their distance from the main bronchus is set to 10 . The parameter $K$ for the KNN classifier is set to 15, and the maximum number of features $F$ for the feature selection process is set to 20 .

An initial set of features containing a total of 147 features are used. These 147 features includes 21 different local descriptors calculated on 7 different scales. The 7 different scales are exponentially distributed within a range from $0.5 \mathrm{~mm}$ to $3.5 \mathrm{~mm}$. This range of scales determines the amount of smoothing applied to the image, or specifically the standard deviation of the Gaussian kernel. ${ }^{20}$ The smoothed version of the image along with its first and second order derivatives are used as local image descriptors. Derivatives are calculated via approximation using finite difference of the smoothed image. Rotational invariant descriptors such as gradient magnitude, Laplacian, and Gaussian curvature are also included. Shape descriptors based on eigenvalues of the Hessian that are commonly used to enhance blob-like, plate-like and tube-like structures ${ }^{21,22}$ are also included into our set of local descriptors, along with the eigenvalues $\lambda_{1}, \lambda_{2}$ and $\lambda_{3}$. The eigenvalues are ordered according to their absolute values such that $\left|\lambda_{1}\right| \geq\left|\lambda_{2}\right| \geq\left|\lambda_{3}\right|$. A detailed list local descriptors used is given in Table 1 .

\subsection{Data}

The proposed segmentation method is tested on 20 low dose CT images from 20 different subjects enrolled in the Danish lung cancer screening trial (DLCST), with a voxel size of $0.78125 \times 0.78125 \times 1 \mathrm{~mm}$ (except for one image that has a voxel size of $0.75 \times 0.75 \times 1 \mathrm{~mm})$. The 20 subjects are selected randomly from the screening study. A ratio of $\mathrm{FEV}_{1} / \mathrm{FVC}$ lower than $70 \%$ is commonly used to determine whether a subject has COPD using lung function test $(\mathrm{LFT}){ }^{2}$ where $\mathrm{FEV}_{1}$ and $\mathrm{FVC}$ are the forced expiratory volume in the first second and forced vital capacity respectively. Among the 20 subjects selected, 12 have $\mathrm{FEV}_{1} / \mathrm{FVC}$ of over $70 \%, 5$ between $70 \%$ to $60 \%$ and 3 lower than $60 \%$. Among the 8 subjects with $\mathrm{FEV}_{1} / \mathrm{FVC}$ lower than $70 \%, 7$ are at stage 1 (mild COPD) and 1 at stage 2 (moderate COPD) according to COPD guidelines from Global Initiative for Chronic Obstructive Lung Disease (GOLD) ${ }^{2}$

A two-fold cross validation experiment is conducted. The 20 subjects are randomly separated into two groups, group A and group B, where group A is used as training set for constructing the classifier that is to be tested on group B and vice-versa. Fig. 2 show the ground truth of the images from group A and group B, obtained using the method described in Sec. 2.4. 
Table 1. Equation used for the calculation of local image descriptors, note that $G_{\sigma}$ is a Gaussian kernel of standard deviation $\sigma$ and the eigenvalues are arranged such that $\left|\lambda_{1}\right| \geq\left|\lambda_{2}\right| \geq\left|\lambda_{3}\right|$.

\begin{tabular}{c|c} 
Description & Equations \\
\hline Convolution with Gaussian & $L=I * G_{\sigma}$ \\
1st order derivatives & $L_{x}, L_{y}, L_{z}$ \\
2nd order derivatives & $L_{x x}, L_{y y}, L_{z z}, L_{x y}, L_{x z}, L_{y z}$ \\
Gradient magnitude & $\sqrt{L_{x}^{2}+L_{y}^{2}+L_{z}^{2}}$ \\
Eigenvalues of Hessian & $\lambda_{1}, \lambda_{2}, \lambda_{3}$ \\
Laplacian & $\lambda_{1}+\lambda_{2}+\lambda_{3}$ \\
Gaussian curvature & $\lambda_{1} \lambda_{2} \lambda_{3}$ \\
Eigen magnitude & $\sqrt{\lambda_{1}^{2}+\lambda_{2}^{2}+\lambda_{3}^{2}}$ \\
Ratios of eigenvalues & $\left|\frac{\lambda_{2}}{\lambda_{1}}\right|,\left|\frac{\lambda_{3}}{\lambda_{1}}\right|, \frac{\left|\lambda_{1}\right|-\left|\lambda_{2}\right|}{\left|\lambda_{1}\right|+\left|\lambda_{2}\right|}, \frac{\left|\lambda_{3}\right|}{\sqrt{\left|\lambda_{1} \lambda_{2}\right|}}$
\end{tabular}

Table 2. TPR, FPR, FDR, NPV and false negative from the KNN classifier and intensity based region growing.

\begin{tabular}{|c|c|c|c|c|c|c|c|c|c|c|}
\hline $\begin{array}{c}\text { Data } \\
\text { set }\end{array}$ & KNN & $\begin{array}{l}\text { Region } \\
\text { growing }\end{array}$ & $\mathrm{KNN}$ & $\begin{array}{l}\text { Region } \\
\text { growing }\end{array}$ & $\mathrm{KNN}$ & $\begin{array}{l}\text { Region } \\
\text { growing }\end{array}$ & $\mathrm{KNN}$ & $\begin{array}{l}\text { Region } \\
\text { growing }\end{array}$ & $\begin{array}{r}\text { False } \\
\text { (pixe } \\
\text { KNN }\end{array}$ & $\begin{array}{l}\text { egative } \\
\text { count) } \\
\text { Region } \\
\text { growing }\end{array}$ \\
\hline A1 & 97.97 & 93.81 & 0.0515 & 0 & 16.45 & 0 & 99.995 & 99.983 & 713 & 2171 \\
\hline $\mathrm{A} 2$ & 98.79 & 92.10 & 0.0232 & 0 & 12.43 & 0 & 99.998 & 99.987 & 265 & 1724 \\
\hline A3 & 99.06 & 97.00 & 0.0403 & 0 & 16.79 & 0 & 99.998 & 99.939 & 138 & 441 \\
\hline $\mathrm{A} 4$ & 96.45 & 100 & 0.0927 & 0.767 & 27.63 & 23.37 & 99.991 & 100 & 857 & 0 \\
\hline A5 & 98.66 & 96.45 & 0.0636 & 0 & 18.31 & 0 & 99.996 & 99.989 & 332 & 877 \\
\hline $\mathrm{A} 6$ & 95.03 & 100 & 0.0994 & 0.1861 & 30.83 & 44.21 & 99.988 & 100 & 1226 & 0 \\
\hline $\mathrm{A} 7$ & 97.96 & 100 & 0.0858 & 0.0909 & 27.91 & 28.66 & 99.995 & 100 & 417 & 0 \\
\hline A 8 & 98.57 & 95.02 & 0.0353 & 0 & 21.47 & 0 & 99.998 & 99.993 & 244 & 847 \\
\hline A9 & 96.38 & 100 & 0.0267 & 0.0025 & 19.98 & 2.17 & 99.996 & 100 & 565 & 0 \\
\hline A10 & 94.59 & 92.61 & 0.0404 & 0 & 11.41 & 0 & 99.982 & 99.976 & 1511 & 2063 \\
\hline B1 & 98.97 & 92.78 & 0.0242 & 0 & 14.08 & 0 & 99.998 & 99.989 & 131 & 920 \\
\hline B2 & 94.79 & 100 & 0.0687 & 24.51 & 18.92 & 98.75 & 99.984 & 100 & 2047 & 0 \\
\hline B3 & 98.85 & 96.55 & 0.0228 & 0 & 8.95 & 0 & 99.997 & 99.992 & 324 & 976 \\
\hline B4 & 87.07 & 100 & 0.0755 & 1.92 & 15.38 & 80.11 & 99.938 & 100 & 7407 & 0 \\
\hline B5 & 99.81 & 95.73 & 0.0379 & 0 & 15.26 & 0 & 99.999 & 99.991 & 39 & 881 \\
\hline B6 & 98.74 & 87.62 & 0.0296 & 0 & 17.81 & 0 & 99.998 & 99.983 & 132 & 1298 \\
\hline B7 & 98.40 & 84.63 & 0.0111 & 0 & 6.67 & 0 & 99.997 & 99.976 & 248 & 2388 \\
\hline B8 & 99.52 & 94.06 & 0.0256 & 0 & 12.59 & 0 & 99.999 & 99.989 & 58 & 712 \\
\hline B9 & 98.82 & 100 & 0.0512 & 0.0278 & 17.22 & 10.03 & 99.997 & 100 & 293 & 0 \\
\hline B10 & 98.81 & 100 & 0.044 & 0 & 17.66 & 6.45 & 99.998 & 100 & 220 & 0 \\
\hline
\end{tabular}

\subsection{Results}

For comparison purpose, results from experiments conducted using an intensity based region growing algorithm is also presented. The optimal threshold for the intensity based region growing algorithm is determined by conducting ROC curve analysis on its performance on the training set.

Surface renderings of the airway segmentation by the proposed method and by the intensity based region growing algorithm are presented in both Fig. 3 and Fig. 4 . True positive rate $(\mathrm{TPR}=\mathrm{TP} / \mathrm{P})$, false positive rate $(\mathrm{FPR}=\mathrm{FP} / \mathrm{N})$, false discovery rate $(\mathrm{FDR}=\mathrm{FP} /(\mathrm{FP}+\mathrm{TP}))$, negative predictive value $(\mathrm{NPV}=\mathrm{TN} /(\mathrm{TN}+\mathrm{FN}))$, and false negative based on the ground truth are also computed from the segmentation results and are shown in Table 2. 

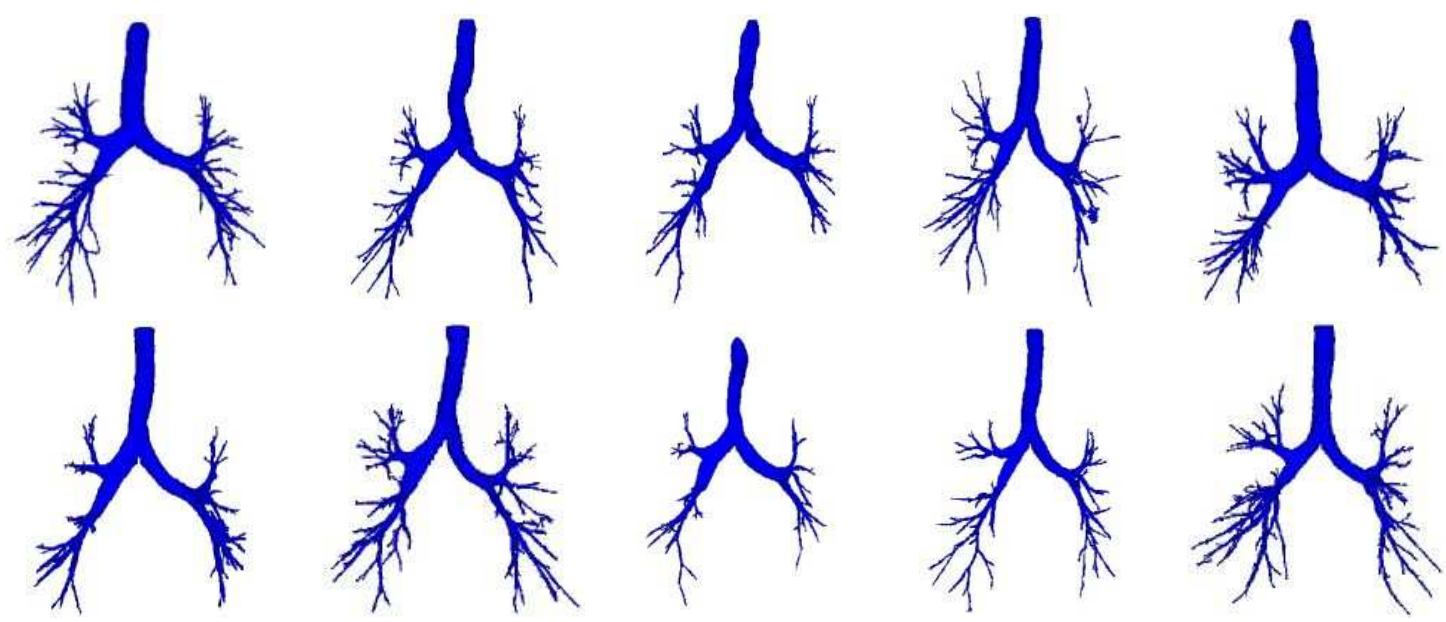

(a) Group A
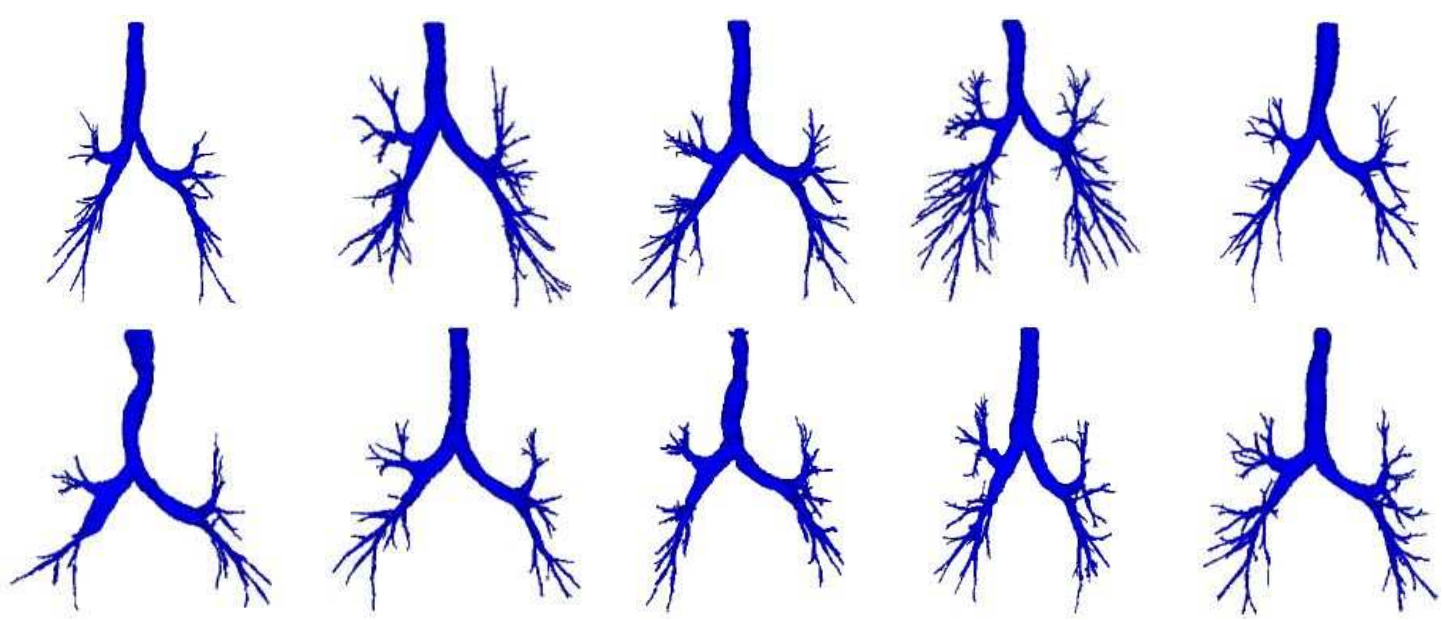

(b) Group B

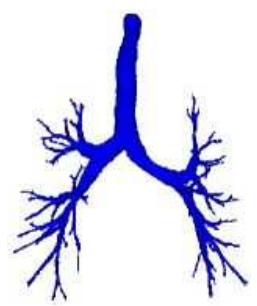

Figure 2. Surface renderings of the ground truth. 

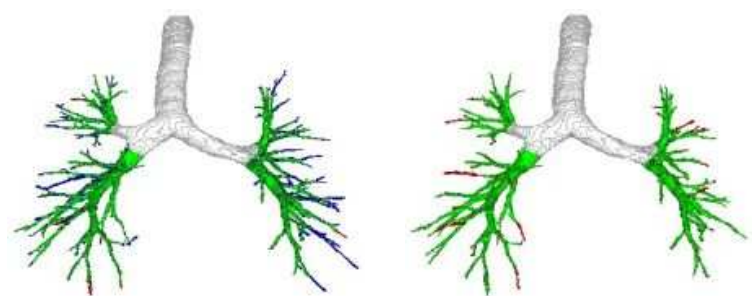

A1
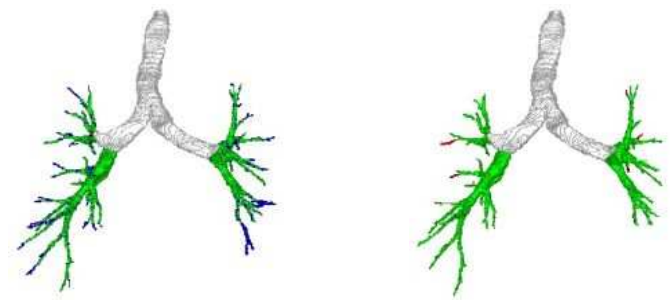

A3

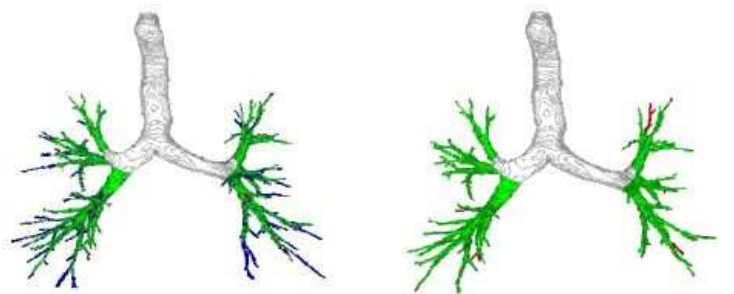

A5
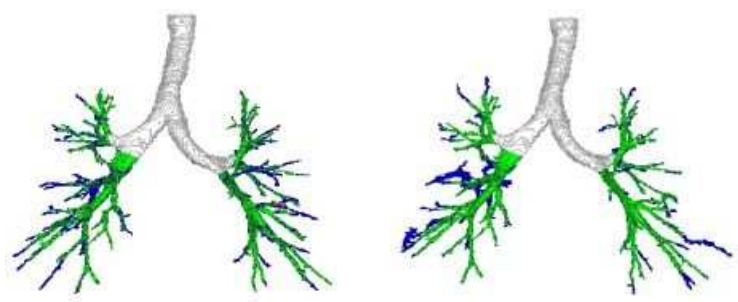

A7

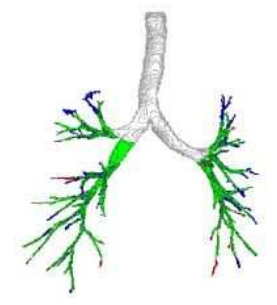

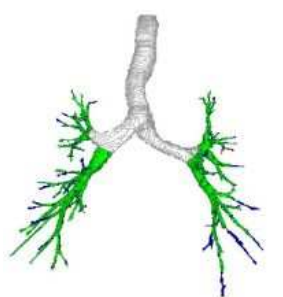

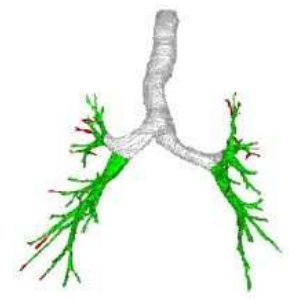

A2
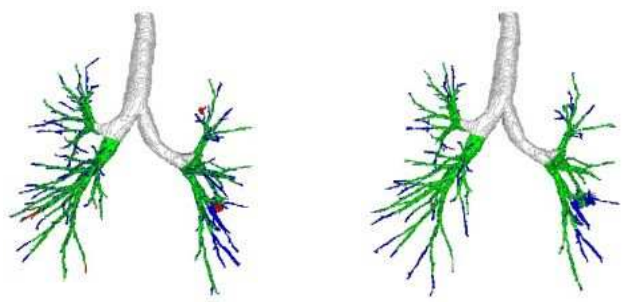

A4
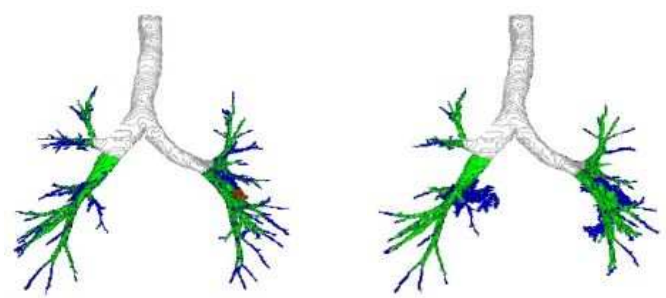

A6
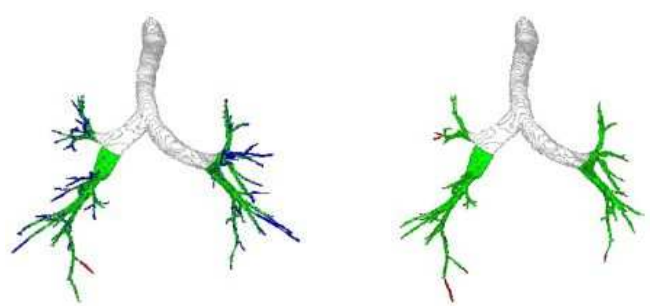

A8
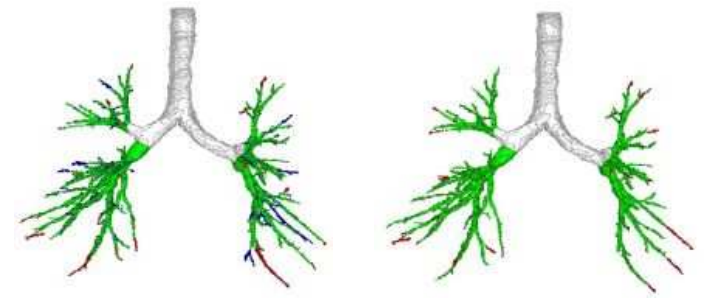

A10

Figure 3. Surface renderings of segmentation results from proposed method (left) and intensity based region growing algorithm (right) with group A as testing. The subtracted trachea and main bronchus are shown in white, true positive are show in green, false positives are shown in blue and false negative in red. 

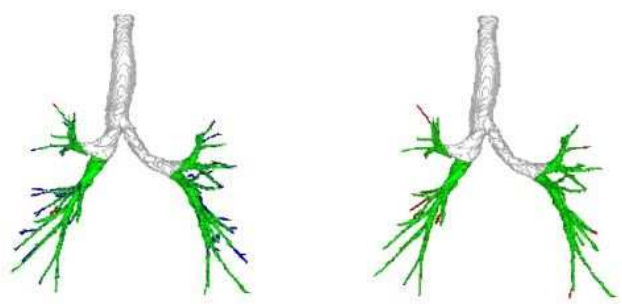

B1

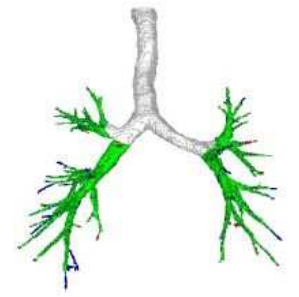

B3
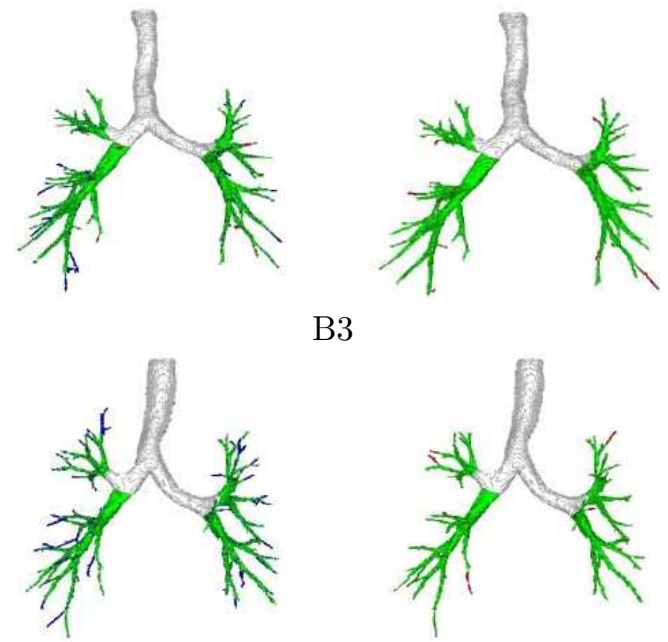

B5
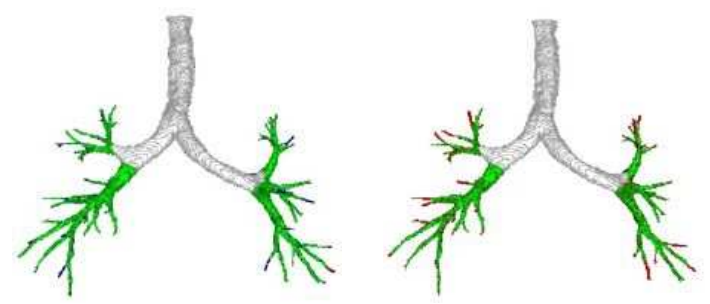

B7
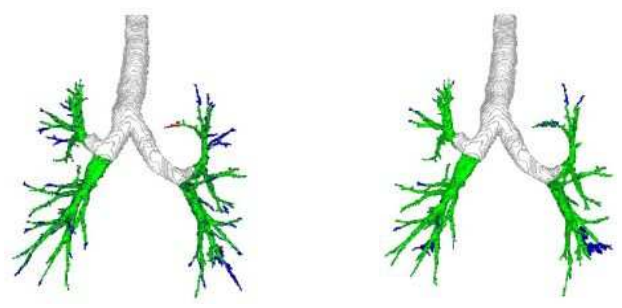

B9
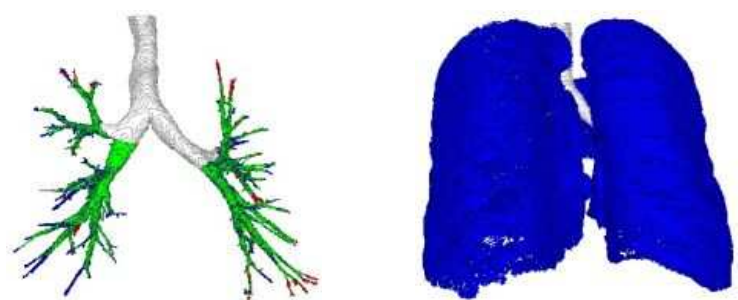

B2
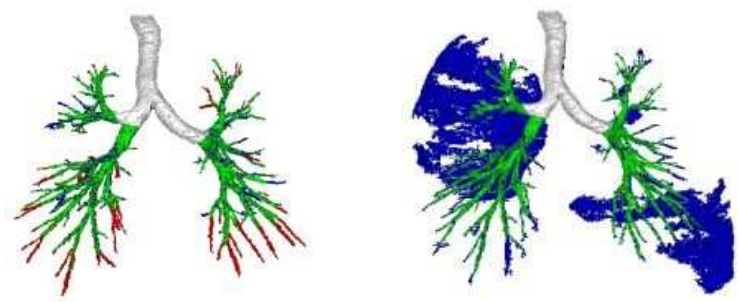

B4
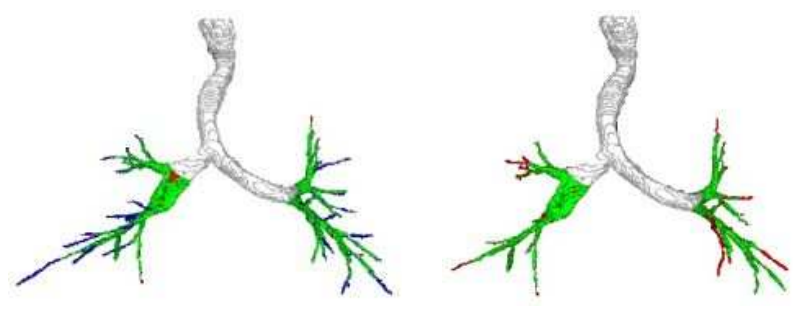

B6
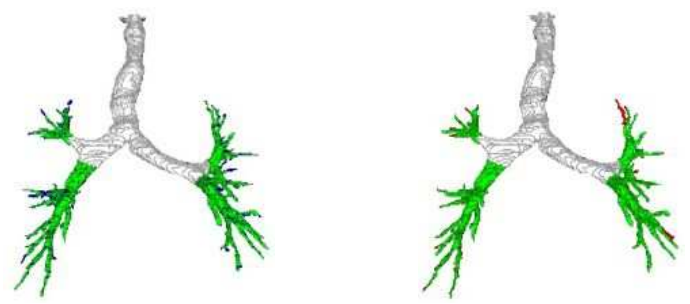

B8
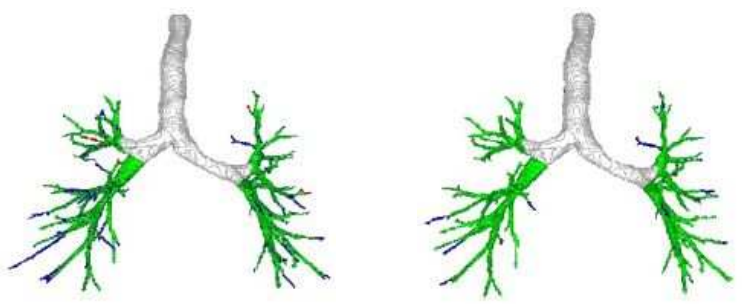

B10

Figure 4. Surface renderings of segmentation results from proposed method (left) and intensity based region growing algorithm (right) with group B as testing. The subtracted trachea and main bronchus are shown in white, true positive are show in green, false positives are shown in blue and false negatives in red. 

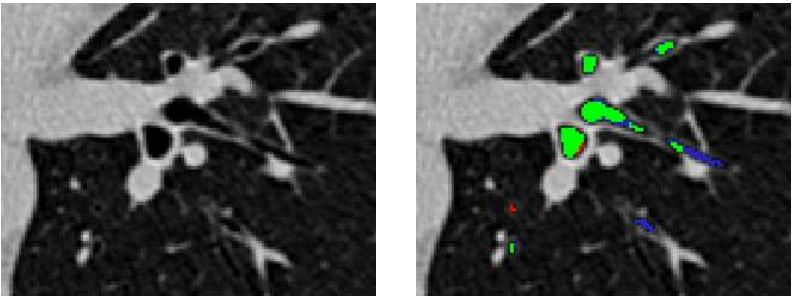

(a)
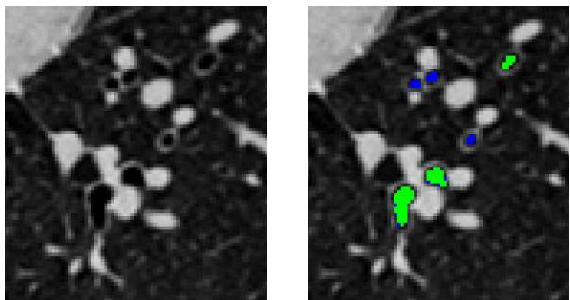

(b)

Figure 5. Axial views, both without (left) and with (right) overlay, showing the new branches found (indicated in blue) by the proposed method. True positives are indicated in green, and false negatives in red.

\section{DISCUSSION}

Fig. 3 and Fig. 4 show that the proposed method generally gives better results as compared to intensity based region growing. The proposed method does not leak significantly in to the lung region and also extracts more airway branches. This is further confirmed by the results in Table 2, where results from the proposed method generally have higher TPR as compared to intensity based region growing. In the cases where intensity based region growing does gives higher TPR, it often results in explosion, which is indicated by relatively high value of FPR and FDR.

It can be observed that results from the proposed method usually have quite a high amount of false positives, as observed from the relatively high FPR and FDR. It should be noted that this is, in many cases, actually preferable and shows one of the main advantages of the proposed method. Due to the imperfect nature of the training data used, there are a significant number of branches missing in the training data. The learning by examples nature of the proposed method would then learn from similar branches successfully labelled in another data set, which makes it possible to pick up such missing branches during the classification process. This can be observed from the surface renderings from Fig. 3 and Fig. 4, where most of the false positives (shown in blue) are actually branches missed by the interactive region growing segmentation. Fig. 5 shows the axial view of examples of the new branches found.

Besides being able to extract new branches that are not in the training data, the proposed method is also capable of correctly segmenting airway branches that are showed as leakage in the ground truth, due to the limitations of the interactive region growing algorithm used. This is shown as false negatives, which may gives an incorrect impression that the proposed method is performing badly. Fig. 6 shows examples of this in the case A4 and A6, where it can be observed that unavoidable leakage from the manual segmentation (indicated by the arrow) is correctly labelled as non-airway by the proposed method.

It is noted that the proposed method performs quite badly for case B4, with only a TPR of $87.07 \%$. The reason for this is because this particular patient has high generation airways that are larger than average. As this is not very common, no similar cases are available in group A. The classifier trained on group A is therefore not capable of handling such special case, which results in rejecting most of the larger than average airways in B4.

\section{CONCLUSIONS}

A voxel classification based airway tree segmentation method, with a KNN classifier trained using sequential forward floating feature selection as the classifier, is presented. Rather than imposing rules to prevent explosion, the proposed method avoids explosion by being able to better classify airways and non-airways using multiple local image descriptors.

Due to the difficulty in obtaining high quality manual annotations of the airway tree, an alternative solution of using 'imperfect' segmentations from an interactive region growing algorithm as a surrogate ground truth is introduced. Application of the voxel classification method is focused on the smaller higher generation of the airways trees. Distance transform is introduced during the sampling of the training points to put a higher emphasis on the higher generation branches. 


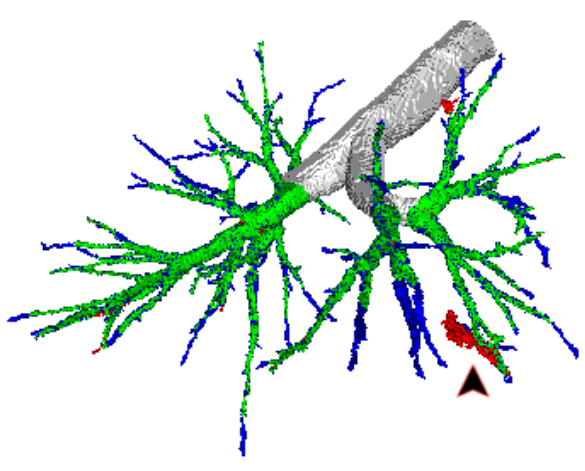

(a)

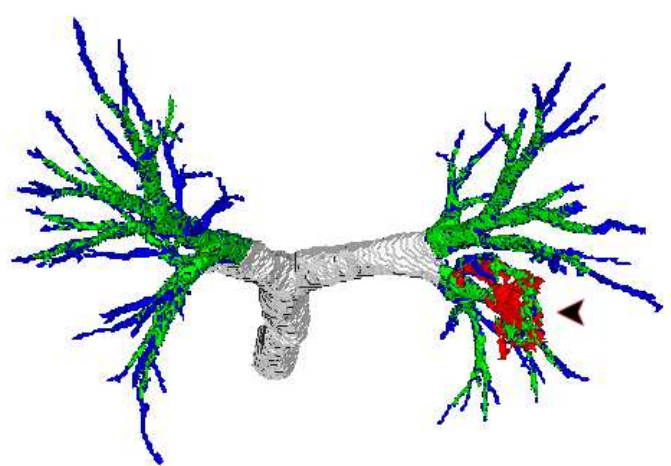

(b)

Figure 6. A better view of case (a) A4 and (b) A6, where a certain degree of unavoidable leakage from the ground truth are labelled correctly as non-airways (pointed by the black arrow and indicated in red) and new branches are discovered.

A total of 20 subjects with varying conditions are tested with the proposed method. In all cases except one, the proposed method performs generally quite well with only a small amount of leakage if there is any. The method is capable of discovering new branches unobtainable by the interactive region growing algorithm used for the training data and can avoid leakage that may be present in the training data due to the limitations of the interactive segmentation algorithm used. Because of these two characteristics, the proposed method is generally capable of producing results that are better than the training data.

\section{ACKNOWLEDGMENTS}

The authors would like to thank Asger Dirksen, Jesper Johannes Holst Pedersen and Haseem Ashraf from Gentofte Hospital and DLCST for providing the CT images and LFT results.

This work is funded by the Danish Council for Strategic Research, under the Programme Commission for Nanoscience and Technology, Biotechnology and IT (NABIIT), and by the Netherlands Organisation for Scientific Research (NWO).

\section{REFERENCES}

1. C. J. Murray and A. D. Lopez, "Evidence-based health policy-lessons from the global burden of disease study," Science 274, pp. 740-743, Nov 1996.

2. K. F. Rabe, S. Hurd, A. Anzueto, P. J. Barnes, S. A. Buist, P. Calverley, Y. Fukuchi, C. Jenkins, R. Rodriguez-Roisin, C. van Weel, J. Zielinski, and Global Initiative for Chronic Obstructive Lung Disease, "Global strategy for the diagnosis, management, and prevention of chronic obstructive pulmonary disease: Gold executive summary," Am J Respir Crit Care Med 176, pp. 532-555, Sep 2007.

3. H. O. Coxson and R. M. Rogers, "Quantitative computed tomography of chronic obstructive pulmonary disease," Acad Radiol 12, pp. 1457-1463, Nov 2005.

4. J. Reinhardt, N. D’Souza, and E. Hoffman, "Accurate measurement of intrathoracic airways," 16(6), pp. 820-827, 1997.

5. Y. Nakano, S. Muro, H. Sakai, T. Hirai, K. Chin, M. Tsukino, K. Nishimura, H. Itoh, P. D. Par, J. C. Hogg, and M. Mishima, "Computed tomographic measurements of airway dimensions and emphysema in smokers. Correlation with lung function," Am J Respir Crit Care Med 162, pp. 1102-1108, Sep 2000.

6. P. Berger, V. Perot, P. Desbarats, J. M. T. de Lara, R. Marthan, and F. Laurent, "Airway wall thickness in cigarette smokers: quantitative thin-section CT assessment," Radiology 235, pp. 1055-1064, Jun 2005.

7. J. M. Kuhnigk, H. Hahn, M. Hindennach, V. Dicken, S. Krass, and H. O. Peitgen, "Lung lobe segmentation by anatomy-guided 3D watershed transform," in Medical Imaging 2003: Image Processing, M. Sonka and J. M. Fitzpatrick, eds., 5032(1), pp. 1482-1490, SPIE, 2003. 
8. C. Wu and G. Agam, "Vessel-based registration with application to nodule detection in thoracic CT scans," in Proc. of SPIE International Symposium on Medical Imaging, 6144, March 2006.

9. K. Mori, J. Hasegawa, J. Toriwaki, H. Anno, and K. Katada, "Recognition of bronchus in three-dimensional X-ray CT images with applications to virtualized bronchoscopy system," in Proc. 13th International Conference on Pattern Recognition, 3, pp. 528-532 vol.3, 1996.

10. A. P. Kiraly, W. E. Higgins, E. A. Hoffman, G. McLennan, and J. M. Reinhardt, "3D human airway segmentation for virtual bronchoscopy," in SPIE Medical Imaging 2002: Physiology and Function from Multidimensional Images, 4683, pp. 16-29, Apr. 2002.

11. T. Schlathölter, C. Lorenz, I. C. Carlsen, S. Renisch, and T. Deschamps, "Simultaneous segmentation and tree reconstruction of the airways for virtual bronchoscopy," Medical Imaging 2002: Image Processing 4684(1), pp. 103-113, SPIE, 2002.

12. T. Kitasaka, K. Mori, Y. Suenaga, J. Hasegawa, and J. Toriwaki, "A method for segmenting bronchial trees from 3D chest X-ray CT images," in MICCAI (2), pp. 603-610, 2003.

13. J. Tschirren, E. Hoffman, G. McLennan, and M. Sonka, "Intrathoracic airway trees: segmentation and airway morphology analysis from low-dose CT scans," Medical Imaging, IEEE Transactions on 24, pp. 15291539, Dec. 2005.

14. H. Singh, M. Crawford, J. P. Curtin, and R. Zwiggelaar, "Automated 3D segmentation of the lung airway tree using gain-based region growing approach," in MICCAI (2), pp. 975-982, 2004.

15. E. van Rikxoort and B. van Ginneken, "A pattern recognition approach to enhancing structures in 3D CT data," in Proc. of SPIE International Symposium on Medical Imaging, J. M. Reinhardt and J. P. W. Pluim, eds., 6144, pp. 569-576, Mar. 2006.

16. R. A. Ochs, J. G. Goldin, F. Abtin, H. J. Kim, K. Brown, P. Batra, D. Roback, M. F. McNitt-Gray, and M. S. Brown, "Automated classification of lung bronchovascular anatomy in CT using AdaBoost," Medical Image Analysis 11, pp. 315-324, June 2007.

17. S. Hu, E. Hoffman, and J. Reinhardt, "Automatic lung segmentation for accurate quantitation of volumetric X-ray CT images," Medical Imaging, IEEE Transactions on 20, pp. 490-498, June 2001.

18. S. Arya, D. M. Mount, N. S. Netanyahu, R. Silverman, and A. Y. Wu, "An optimal algorithm for approximate nearest neighbor searching fixed dimensions," J. ACM 45(6), pp. 891-923, 1998.

19. P. Pudil, J. Novovičová, and J. Kittler, "Floating search methods in feature selection," Pattern Recogn. Lett. 15(11), pp. 1119-1125, 1994.

20. L. M. J. Florack, B. M. t. Haar Romeny, J. J. Koenderink, and M. A. Viergever, "Scale and the differential structure of images," 10, pp. 376-388, July/August 1992.

21. A. F. Frangi, W. J. Niessen, K. L. Vincken, and M. A. Viergever, "Multiscale vessel enhancement filtering," in Medical Image Computing and Computer-Assisted Interventation - MICCAI '98, pp. 130+, 1998.

22. R. Wiemker, T. Bulow, and T. Blaffert, "Unsupervised extraction of the pulmonary interlobar fissures from high resolution thoracic CT data," International Congress Series 1281, pp. 1121-1126, May 2005. 